REPLY

\title{
Heritable contributions versus genetic architecture
}

Nicholas J. Timpson, Celia M. T. Greenwood, Nicole Soranzo, Daniel J. Lawson and J. Brent Richards

We read with interest the correspondence by Evans and Keller (Using partitioned heritability methods to explore genetic architecture. Nat. Rev. Genet. https://doi.org/10.1038/ nrg.2018.6) ${ }^{1}$ on our recent Review (Genetic architecture: the shape of the genetic contribution to human traits and disease. Nat. Rev. Genet. 19, 110-124 (2018))2. Heritability estimation and partition is indeed an interesting aggregate approach for the analysis of genetic contributions to phenotypic variability and one that is afforded by a genetic contribution to variance in traits of interest. Heritability analyses such as those described are not designed to dissect a complete genetic architecture. It is important to note that although partition or specifically designed heritability analyses can give insight into the overall genetic contribution to a trait or disease outcome, it is based on a prior for that analysis and on the overall genetic contribution given that prior (for example, analysis by minor allele frequency). Especially when based on chip genotype data, these types of analyses are informative but do not provide a complete portrait of genetic architecture, which can vary for any given heritability. We recognize the utility of novel approaches to the analysis of heritable contributions and add that other approaches with interesting study designs, for example, the quantification of maternal heritable contributions to offspring phenotype (for a description of this approach see REF. ${ }^{3}$; for an example of its application see REF. ${ }^{4}$ ) should be considered for partitioning heritable contributions.
Nicholas J. Timpson ${ }^{1 *}$, Celia M. T. Greenwood ${ }^{2,3}$, Nicole Soranzo ${ }^{4,5}$, Daniel J. Lawson ${ }^{7}$ and J. Brent Richards $3,6,7 *$

${ }^{1}$ MRC Integrative Epidemiology Unit, School of Social and Community Medicine, University of Bristol, Bristol, UK.

${ }^{2}$ Department of Oncology, McGill University, Montreal, Quebec, Canada.

${ }^{3}$ Departments of Human Genetics and Epidemiology, Biostatistics and Occupational Health, McGill University, Montreal, Quebec, Canada.

${ }^{4}$ The Wellcome Trust Sanger Institute, Hinxton, Cambridge, UK.

${ }^{5}$ Department of Haematology, University of Cambridge, Cambridge, UK.

${ }^{6}$ Department of Medicine, Jewish General Hospital, Lady Davis Institute, McGill University, Montreal, Quebec, Canada.

${ }^{7}$ Department of Twin Research \& Genetic Epidemiology, King's College London, London, UK.

*e-mail:n.j.timpson@bristol.ac.uk; brent.richards@mcgill.ca

doi:10.1038/nrg.2018.7 Published online 14 Feb 2018

1. Evans, L. M. \& Keller, M. C. Using partitioned heritability methods to explore genetic architecture. Nat. Rev. Genet. https://doi.org/10.1038/nrg.2018.6 (2018).

2. Timpson, N. J. et al. Genetic architecture: the shape of the genetic contribution to human traits and disease. Nat. Rev. Genet. 19, 110-124 (2018).

3. Eaves, L. J. et al. Resolving the effects of maternal and offspring genotype on dyadic outcomes in genome wide complex trait analysis ("M-GCTA"). Behav. Genet. 44, 445-455 (2014).

4. Horikoshi, M. et al. Genome-wide associations for birth weight and correlations with adult disease. Nature 538, 248-252 (2016).

Competing interests

The authors declare no competing interests. 